\title{
Response to Dawson and Arkes
}

The Authors Reply:- Drs. Dawson and Arkes are to be commended on their detailed analysis of our paper. However, as our response indicates, their analysis fails to affect the interpretation of the data we reported.

Drs. Dawson and Arkes express concern that our study did not provide the opportunity for residents to list a differential diagnosis for the patient's chest pain and that we interpreted cross-sectional data as if they were longitudinal data. What they fail to realize is that in the emergency setting, even with a differential diagnosis in mind, a physician must ultimately make a decision as to whether or not to recommend thrombolysis. At least five studies (not conducted by any of us) have demonstrated that African-American patients are less likely to receive thrombolysis under this circumstances. Our concern was not with the role of race in the recommendation of thrombolysis. Rather, we were interested in the degree to which racial bias as measured by a test of implicit race attitude (the IAT) would predict physicians' recommendation of thrombolysis to black versus white patients. As is routine in such studies (and in the situations physicians commonly face), we designed the vignette to be ambiguous so as to test the direction of decisions that individual doctors would follow. Indeed, we found that IAT scores are correlated with treatment decisions, and that this correlation is opposite for black and white patients. Additionally, given that these are cross-sectional data, we carefully offered no causal claims in the article. Nor do we make any claim regarding the mechanism that produces this relationship.

Regarding the use of terms such as "increasing time for association," "racial preference," "racial bias," and "pro-white/ pro-black scores," we agree that these do not represent a sequence of synonyms. However, these terms are appropriate for the context in which they appear in the manuscript and did not seem to mislead our own internal reviewers, the JGIM peer reviewers, or the editors.

Regarding our analyses, we understand why Dawson and Arkes were tempted to interpret the interaction plot the way they did. Across social and medical science it is common for scientists to graph interactions with plots depicting both the interaction variance and the main effect variance even though

Published online October 29, 2008 the statistical test of the interaction refers only to the pure (i.e., unique) interaction variance. As such, one is tempted to interpret the means (in the case of a bar graph) or the data points on a line graph (i.e., the four data points the authors refer to and for which a bar graph was provided).

However, it is a mistake to interpret the means as part of the interaction when one is interested purely in the nature of the interaction. Interaction plots such as these are not pure interaction plots; they contain variance from main effects. To the extent that main effects are non-zero, an interaction graph will not appear to be a crossover interaction. Any pure interaction looks exactly the same; as a cross-over pattern (see Ralph Rosnow and Robert Rosenthal, 1991). ${ }^{1}$ Thus, a reinterpretation of our result on this basis is incorrect. Our data show that as physicians' bias against blacks increased, recommendations decreased. This was the reverse for white patients. This was our modest conclusion, and the one we stand by. We cannot abide by Dawson and Arkes' suggestion to interpret the behavior of doctors at specific points along the continuum.

Finally, due to space limitations, we did not include a detailed discussion of alternative hypotheses. This limitation cannot affect the integrity of the study itself, and we appreciate that Dawson and Arkes have provided these on our behalf.

Alexander R. Green, MD, MPH, The Disparities Solutions Center, Massachusetts General Hospital, Harvard Medical School, 50 Staniford Street - Suite 901, Boston, MA 02114, USA (e-mail: argreen@partners.org).

\section{REFERENCE}

1. Rosnow RL, Rosenthal R. If you're looking at the cell means, you're not looking at only the interaction (unless all main effects are zero). Psychol Bull. 1991;110:574-6.

$\mathrm{J}$ Gen Intern Med 24(1):141

DOI: $10.1007 / \mathrm{s} 11606-008-0822-7$

() Society of General Internal Medicine 2008 